\title{
Comparing Preventable Acute Care Use of Rural Versus Urban Americans: an Observational Study of National Rates During 2008-2017
}

\author{
Kenton J. Johnston, $P h D^{7}$ (D) , Hefei Wen, $P h D^{2}$, Ameya Kotwal, BS ${ }^{3}$, and Karen E. Joynt \\ Maddox, MD MPH ${ }^{4}$
}

\begin{abstract}
'Department of Health Management and Policy and Center for Outcomes Research, College for Public Health and Social Justice, Saint Louis University 3545 Lafayette Ave., Room 362, St. Louis, MO, USA; ${ }^{2}$ Division of Health Policy and Insurance Research, Department of Population Medicine, Harvard Medical School \& Harvard Pilgrim Health Care Institute 401 Park Drive, Suite 401 East, Boston, MA, USA; ${ }^{3}$ Department of Health Management and Policy, College for Public Health and Social Justice, Saint Louis University 3545 Lafayette Ave, St. Louis, MO, USA; ${ }^{4}$ Cardiovascular Division, Washington University School of Medicine 660 S. Euclid Ave, CB 8086, St. Louis, MO, USA.
\end{abstract}

BACKGROUND: Rural Americans have less access to care than urban Americans. Preventable acute care use is a marker of unmet ambulatory healthcare needs, but little is known about how such utilization has differed between rural and urban areas over time.

OBJECTIVE: Compare preventable emergency department (ED) visit and hospitalization rates among rural versus urban residents over the past decade.

DESIGN: Observational study using a validated algorithm to compute age-sex-adjusted rates per 100,000 individuals of preventable ED visits and hospitalizations. Differences in overall, annual, and condition-specific rates for rural versus urban residents were assessed and linear regression was used to assess 10-year trends.

SETTING: Nationwide Emergency Department Sample, National Inpatient Sample, and US Census, 2008-2017.

PARTICIPANTS: US adults, an annual average of 241.3 million individuals.

MEASUREMENTS: Preventable ED visits and hospitalizations.

RESULTS: Compared to urban residents, rural residents had $45 \%$ higher rates of preventable ED visits in 2008 (3003 vs. 2070 per 100,000, adjusted difference [AD]: 933; 95\% CI: 928-938) and 44\% higher rates of preventable ED visits in 2017 (3911 vs. 2708 per 100,000, AD: 1202; 95\% CI: 1196-1208). Rural residents had 26\% higher rates of preventable hospitalizations in 2008 (2104 vs. 1666 per 100,000, AD: 439; 95\% CI: 434-443) and 13\% higher rates in 2017 (1634 vs. 1440 per 100,000, AD: 194; 95\% CI: 190-199). Preventable ED visits increased more in absolute terms in rural versus urban residents, but the percentage increase was similar (30\% vs. 31\%) because rural residents started at a higher baseline. Preventable hospitalizations decreased at a faster rate (22\% vs. 14\%) among rural versus urban residents.

LIMITATIONS: Observational study; unable to infer causality.

CONCLUSIONS: Rural disparities in acute care use are narrowing for preventable hospitalizations but have persisted for all preventable acute care use, suggesting

Received September 29, 2020

Accepted December 20, 2020

Published online January 28, 2021 unmet demand for high-quality ambulatory care in rural areas.

J Gen Intern Med 36(12):3728-36

DOI: $10.1007 / \mathrm{s} 11606-020-06532-4$

(C) Society of General Internal Medicine 2021

\section{INTRODUCTION}

Rural Americans have less access to care and experience poorer health outcomes than their urban counterparts. Over the past several decades, rural areas have experienced persistent shortages in primary care physicians (PCPs) ${ }^{1}$ and specialists. ${ }^{2}$ Despite higher rates of chronic disease and complications in rural areas, ${ }^{3-5}$ rural residents with chronic conditions have been less likely to receive ambulatory care to manage their conditions, $2,6,7$ more likely to receive lower quality of ambulatory care, and less likely to receive recommended preventive screenings. ${ }^{3,8,9}$ Prior research indicates rural physician shortages help explain the growing gap in mortality and hospitalization rates between rural and urban residents, especially for those with chronic conditions. ${ }^{1,2,10-12}$

A key population health indicator for tracking unmet healthcare needs is acute care use in the form of emergency department (ED) visits and hospitalizations for conditions such as pneumonia, asthma, diabetes, and heart failure. ${ }^{13}$ These acute care events are considered by the Agency for Healthcare Research and Quality (AHRQ) to be potentially preventable if patients have access to timely and high-quality outpatient care. ${ }^{14-16}$ Treating preventable complications of these conditions in the acute care setting costs the health system billions of dollars annually that could be better spent on preventive care in the ambulatory setting. ${ }^{17,} 18$

Past studies indicate that all-cause ED visit and hospitalization rates among rural residents are 50\% and 22\% higher than those of urban residents, respectively. ${ }^{19-21}$ Furthermore, all-cause ED visits are on the rise in both rural and urban areas. ${ }^{19,21}$ However, research focusing on preventable hospitalizations and ED visits (including observation stays) in rural versus urban areas is 
lacking. It is important to understand the nature of these rates and trends over the past decade as they signal unmet demand for preventive and ambulatory care as well as disparities in health outcomes due to preventable complications of disease.

Our objective was to compare preventable ED visit and hospitalization rates among rural versus urban residents nationally over the past decade. To do this, we focused on two research questions. First, did differences exist in population rates and trends in preventable ED visits between rural and urban adult Americans over the past decade and if so, what conditions explained the largest portion of these differences? Second, did differences exist in rates and trends in preventable hospitalizations between rural and urban adult Americans over the past decade, and if so, what conditions explained the largest portion of these differences?

\section{METHODS}

This study was approved by the Saint Louis University institutional review board, waiving participant written informed consent due to the deidentified retrospective nature of the data.

\section{Data Sources and Study Population}

We used data from the Nationwide Emergency Department Sample (NEDS), National Inpatient Sample (NIS), and the US Census to conduct a 10-year observational study (2008-2017) of hospital discharges and ED visits for adults at general acute care hospitals in the USA. The NEDS and the NIS are the largest publicly available all-payer hospital databases in the USA and approximate a nationally representative $20 \%$ stratified sample of all ED visits and inpatient discharges at US hospitals. ${ }^{22-24} \mathrm{We}$ obtained annual US population denominator estimates from AHRQ that were derived from US Census Bureau estimates and summed over county populations. ${ }^{25}$ We used the NEDS and NIS data to identify preventable ED visits and hospitalizations in the numerator patient population, and then used the population denominator estimates to calculate population rates.

Our study numerator population included all hospital discharge records for patients ages 18 and over with identifiable preventable ED visits or preventable hospitalizations in the NEDS and NIS data for the years 2008-2017. We used the NEDS and NIS discharge weights to generate national estimates of the numerators from the $20 \%$ annual samples. Our study denominator population included all US adults counted by the US Census and included in the postcensal estimates for the years 2008-2017.

\section{Study Variables}

Our outcome variables were population rates of preventable ED visits and hospitalizations. We identified preventable ED visits and hospitalizations using the International Classification of Diseases (ICD) diagnosis codes listed on hospital discharge records for ED visits and inpatient stays in the
NEDS and NIS using the Prevention Quality Indicators algorithm provided by AHRQ. ${ }^{14}$ This algorithm was originally designed to identify preventable hospitalizations for adults due to acute and chronic diagnoses commonly treated in the ambulatory care setting. ${ }^{26}$ However, it has since been expanded to also identify preventable ED visits using the same set of ICD codes. ${ }^{13,}{ }^{27}$ We included all ED visits flagged by the algorithm in our preventable ED visit count, including treatand-release visits and observation stays, except for visits that resulted in admission to the hospital (which are included in the inpatient algorithm). We included all inpatient hospitalizations flagged by the algorithm in our preventable hospitalization count. We counted total, acute, chronic, and condition-specific preventable ED visits and hospitalizations for the following conditions: dehydration, pneumonia, urinary tract infection, diabetes, chronic obstructive pulmonary disease (COPD)/asthma, hypertension, and heart failure.

Next, we identified the geographic location of patients' county of residence as listed on the ED visit and inpatient hospitalization discharge records using the recorded Office of Management and Budget (OMB) county classification codes. Specifically, we used the rural-urban classification schema deployed by the National Center for Health Statistics in 2013 to create two categories ${ }^{1}$ : rural (non-metropolitan counties of less than 50,000 population) and $^{2}$ urban (metropolitan counties with populations of 50,000 or more). ${ }^{28}$ This schema has been previously employed in other studies comparing rural and urban rates, in addition to the National Center for Health Statistics. ${ }^{1,29,30}$ We aggregated our numerator data for total preventable ED visits and hospitalizations (separately) by these two geographic categories. We then applied this same schema to the US Census denominator population by county and summed the population estimates across rural and urban counties to aggregate the denominator data. Finally, we used both the numerator and denominator data to compute national adult population rates of preventable ED visits and hospitalizations per 100,000 rural and urban residents annually over the period 2008-2017. We computed overall, annual, and condition-specific rates.

We also obtained patient age, sex, and comorbidities (Elixhauser Comorbidity Index) from the NEDS and NIS hospital discharge records, as well as age- and sex-specific county population estimates from the US Census data.

\section{Age-Sex Adjustment of Outcome Rates}

Because the rural and urban US populations have different age and sex distributions, we age- and sex-adjusted the preventable ED visit and hospitalization outcome rates. This is the standard approach recommended by AHRQ for computing population-level rates in different geographic regions. ${ }^{14} \mathrm{We}$ computed the adjustment for each year using the direct method of standardization and applying the US national population estimates by age and sex from the US Census data as the reference population. 


\section{Statistical Analysis}

We conducted three primary sets of analyses and two sets of sensitivity analyses. We did these analyses separately for preventable ED visits versus hospitalizations. First, we assessed differences in total, acute, chronic, and conditionspecific outcome rates at the beginning (2008) and end (2017) of the 10-year period for rural versus urban residents, using the normal approximation to the binomial distribution to create 95\% confidence intervals (CIs). Second, we assessed the 10year trend in the total outcome rates for rural and urban residents as the percentage change from 2008 to 2017, the average annual change as the slope coefficient from the least squares regression of the outcome rates on calendar year, and the significance as the $t$ test statistic for calendar year from the least squares regression. Third, to identify the conditions explaining differences in the total outcome rates, we assessed condition-specific outcome rates over the entirety of the 10year period, again using the normal approximation to the binomial distribution to test differences in rates between rural and urban residents.

In sensitivity analyses, we assessed the 10-year trends in the total, acute, chronic, and condition-specific outcome rates for rural and urban residents as the percentage rather than absolute change from 2008 to 2017 and tested for significance in trend using the $t$ test from the slope coefficient of the least squares regression of the outcome rates on calendar year. In addition, we also described the actual unadjusted age, sex, and comorbidity distribution of the numerator patient populations and further assessed the unadjusted total outcome rates overall and by age and sex at the beginning (2008) and end (2017) of the 10 -year period for rural versus urban residents. This approach allowed us to discern whether age-sex adjustment made much relative difference in rural versus urban rates and to assess coding intensity in rural and urban areas over time in the diagnosis-based Elixhauser Comorbidity Index.

We performed analyses using SAS software, version 9.4, and Microsoft Excel, 2016.

\section{Role of the Funding Source}

Saint Louis University purchased and provided access to the data used in this study. The funder had no role in the design and conduct of the study; collection, management, analysis, or interpretation of the data; preparation, review, or approval of the manuscript; or decision to submit the manuscript for publication.

\section{RESULTS}

\section{Description of Study Population}

The study included an annual average of 241.3 million US adults, of whom $15.6 \%$ and $84.4 \%$ lived in rural and urban areas, respectively (Appendix Table 1). There was a $7.6 \%$ decline in the population living in rural areas and a $13.1 \%$ increase in the population living in urban areas over the 10year study period (Appendix Table 2).

\section{Preventable Emergency Department Visit Rates and Trends for Rural Versus Urban Residents}

The study numerator population for preventable ED visits comprised a total of 60.9 million visits, of which $21.2 \%$ and $78.8 \%$ were for patients living in rural and urban areas, respectively (Appendix Table 1). Raw visit totals increased by $22.0 \%$ and $47.7 \%$ over the 10 -year period for patients living in rural and urban areas, respectively (Appendix Table 2). Average comorbidity counts among patients with ED visits in all areas increased substantially across the 10 -year period.

Age- and sex-adjusted preventable ED visit rates were 3003 and 2070 per 100,000 for rural versus urban residents in 2008, respectively, an absolute difference of 933 (95\% CI: 928-938) and percentage difference of $45 \%$ (Table 1). By 2017, total preventable ED visit rates were 3911 and 2708 per 100,000 for rural versus urban residents, respectively, an absolute difference of 1202 (95\% CI: 1196-1208) and percentage difference of $44 \%$. Total preventable ED visit rates rose by $30 \%$ and $31 \%$ for rural and urban residents over the decade ( $p<.001$, Fig. 1 ), respectively, with rural rates $38 \%$ to $60 \%$ higher than urban rates throughout the period $(p<.001)$. Preventable ED visits increased more in rural versus urban residents (908 vs. 638 absolute increase in rates over the 10 years), but the percentage increase was similar (30\% vs. $31 \%$ ) because rural residents started at a higher baseline.

Patterns were similar among the subgroups of both acute and chronic preventable ED visits, as well as all conditionspecific visits except for asthma in younger adults (Table 1). The primary conditions driving higher preventable ED visit rates among rural versus urban residents throughout the decade were urinary tract infections, chronic obstructive pulmonary disease/asthma, pneumonia, and dehydration $(p<.001$, Fig. 2). Preventable ED visit rates increased the most for uncontrolled diabetes in all areas over the 10-year period ( $p$ $<.001$, Appendix Table 1). This increase was primarily due to the switch from the ICD-9-CM to ICD-10-CM diagnosis coding system and the increased use of the ICD-10-CM codes for uncontrolled diabetes in 2015-2017. In sensitivity analyses, results were similar when comparing unadjusted preventable ED visit rates across all areas (Appendix Table 2).

\section{Preventable Hospitalization Rates and Trends for Rural Versus Urban Residents}

The study numerator population for preventable hospitalizations comprised a total of 37.9 million hospitalizations, of which $20.5 \%$ and $79.5 \%$ were for patients living in rural and urban areas, respectively (Appendix Table 3). These raw hospitalization totals decreased by $28.9 \%$ and $1.6 \%$ over the 10 -year period for patients living in rural and urban areas, respectively (Appendix Table 4). Average comorbidity counts 
Table 1 A Comparison of Rural Versus Urban Preventable ED Visit Rates at the Beginning and End of the Decade

\begin{tabular}{|c|c|c|c|c|c|c|c|}
\hline & \multicolumn{4}{|c|}{2008 rates per 100,000 adults $^{b}$} & \multicolumn{3}{|c|}{2017 rates per 100,000 adults ${ }^{b}$} \\
\hline & Rural & Urban & Difference & $(95 \% \mathrm{CI})^{\mathrm{c}}$ & Rural & Urban & $(95 \% \mathrm{CI})^{\mathrm{c}}$ \\
\hline Adult population in millions, $N^{\mathrm{a}}$ & 38.9 & 191.1 & - & & 35.9 & 216.1 & - \\
\hline Males & 19.2 & 92.4 & - & & 17.9 & 104.9 & - \\
\hline Females & 19.7 & 98.8 & - & & 18.0 & 111.2 & - \\
\hline Ages 18-39 & 13.6 & 77.6 & - & & 12.3 & 84.9 & - \\
\hline Ages $40-64$ & 17.3 & 82.7 & - & & 14.9 & 89.1 & - \\
\hline Ages $65+$ & 8.0 & 30.8 & - & & 8.7 & 42.1 & - \\
\hline All preventable ED visits & 3003 & 2070 & $933(928,938)$ & & 3911 & 2708 & $1202(1196,1208)$ \\
\hline Acute preventable ED visits & 1687 & 1096 & $591(587,595)$ & & 2127 & 1415 & $712(708,717)$ \\
\hline Dehydration & 234 & 153 & $82(80,83)$ & & 328 & 198 & $130(129,132)$ \\
\hline Community-acquired pneumonia & 288 & 182 & $107(105,108)$ & & 331 & 216 & $114(113,116)$ \\
\hline Urinary tract infection & 1165 & 762 & $403(400,406)$ & & 1469 & 1001 & $468(464,471)$ \\
\hline Chronic preventable ED visits & 1315 & 974 & $342(338,345)$ & & 1783 & 1293 & $490(486,494)$ \\
\hline Diabetes short-term complications & 9 & 5 & $4(4,4)$ & & 13 & 6 & $7(7,7)$ \\
\hline Diabetes long-term complications & 148 & 113 & $36(34,37)$ & & 66 & 59 & $7(6,7)$ \\
\hline COPD/asthma & 492 & 338 & $154(152,156)$ & & 617 & 372 & $245(243,247)$ \\
\hline Hypertension & 288 & 244 & $44(42,46)$ & & 501 & 393 & $108(106,110)$ \\
\hline Heart failure & 113 & 59 & $54(53,55)$ & & 160 & 100 & $59(58,61)$ \\
\hline Uncontrolled diabetes & 25 & 15 & $10(9,10)$ & & 290 & 225 & $65(63,67)$ \\
\hline Asthma in younger adults & 241 & 200 & $41(39,42)$ & & 137 & 138 & $-1(-2,1)$ \\
\hline
\end{tabular}

${ }^{a}$ Denominator population supplied by AHRQ and derived from the US Census Bureau postcensal estimates summed over county populations for rural (metropolitan area) and urban (city or metropolitan area $\geq 50,000$ population) counties

${ }^{b}$ Weighted estimates from the Nationwide Emergency Department Sample (NEDS) for all patients with at least 1 of the AHRQ Prevention Quality Indicator (Preventable) ED visits that did not result in an inpatient stay. Using the NEDS discharge weights to generate a national estimate by the rural and urban county categories above as noted for patient location on the discharge records. Rates were age-sex-adjusted using annual US Census data on the national US adult population with the direct method of standardization

${ }^{c} 95 \%$ confidence intervals (CIS) calculated using the normal approximation to the standard normal distribution

increased substantially for admitted patients in all areas across the 10-year period.

Age- and sex-adjusted preventable hospitalization rates were 2104 and 1666 per 100,000 for rural versus urban residents in 2008, respectively, an absolute difference of 439 (95\% CI: $434-443$ ) and percentage difference of $26 \%$ (Table 2). By 2017, total preventable hospitalization rates were 1634 and 1440 per 100,000 for rural versus urban residents, respectively, an absolute difference of 194 (95\% CI: 190-199) and percentage difference of $13 \%$. Total preventable hospitalization rates fell by $22 \%$ and $30 \%$ for rural and urban residents over the study period ( $p<.001$, Fig. 3 ), respectively, with rural rates $13 \%$ to $35 \%$ higher than urban rates throughout $(p<.001)$.

Patterns were similar among the subgroups of both acute and chronic preventable hospitalizations, as well for the conditions of dehydration, pneumonia, urinary tract infection, diabetes short-term complications, and COPD/asthma (Table 2). The primary conditions driving higher preventable hospitalization rates among rural versus urban residents throughout the decade were pneumonia, COPD/asthma, urinary tract infections, and dehydration; these were also the most common conditions $(p<.001$, Fig. 4$)$. The exception to this

10-Year Trend in Rural Versus Urban Preventable Emergency Department Visits: 2008-2017 Per 100,000 Adult Population USA, Risk Adjusted for Age and Sex

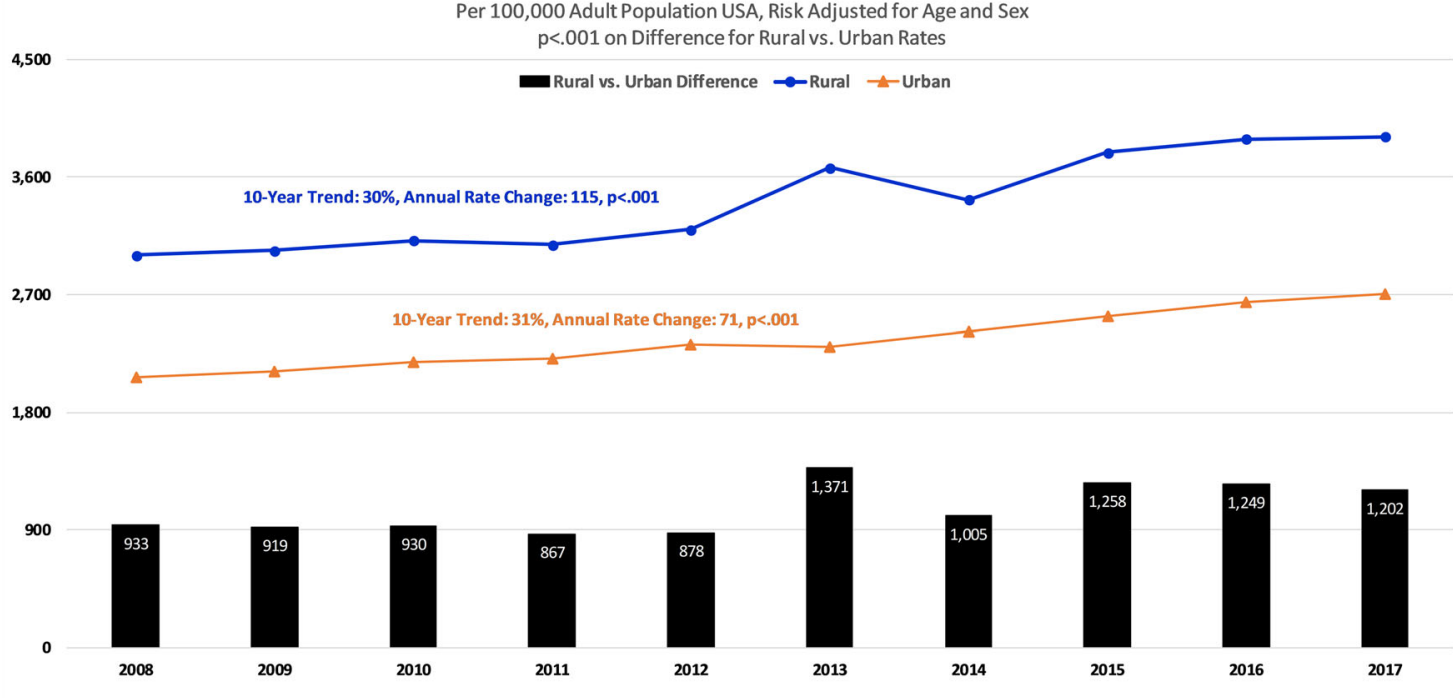

Figure 1 Ten-year trend in rural versus urban preventable emergency department visits: $2008-2017$. 


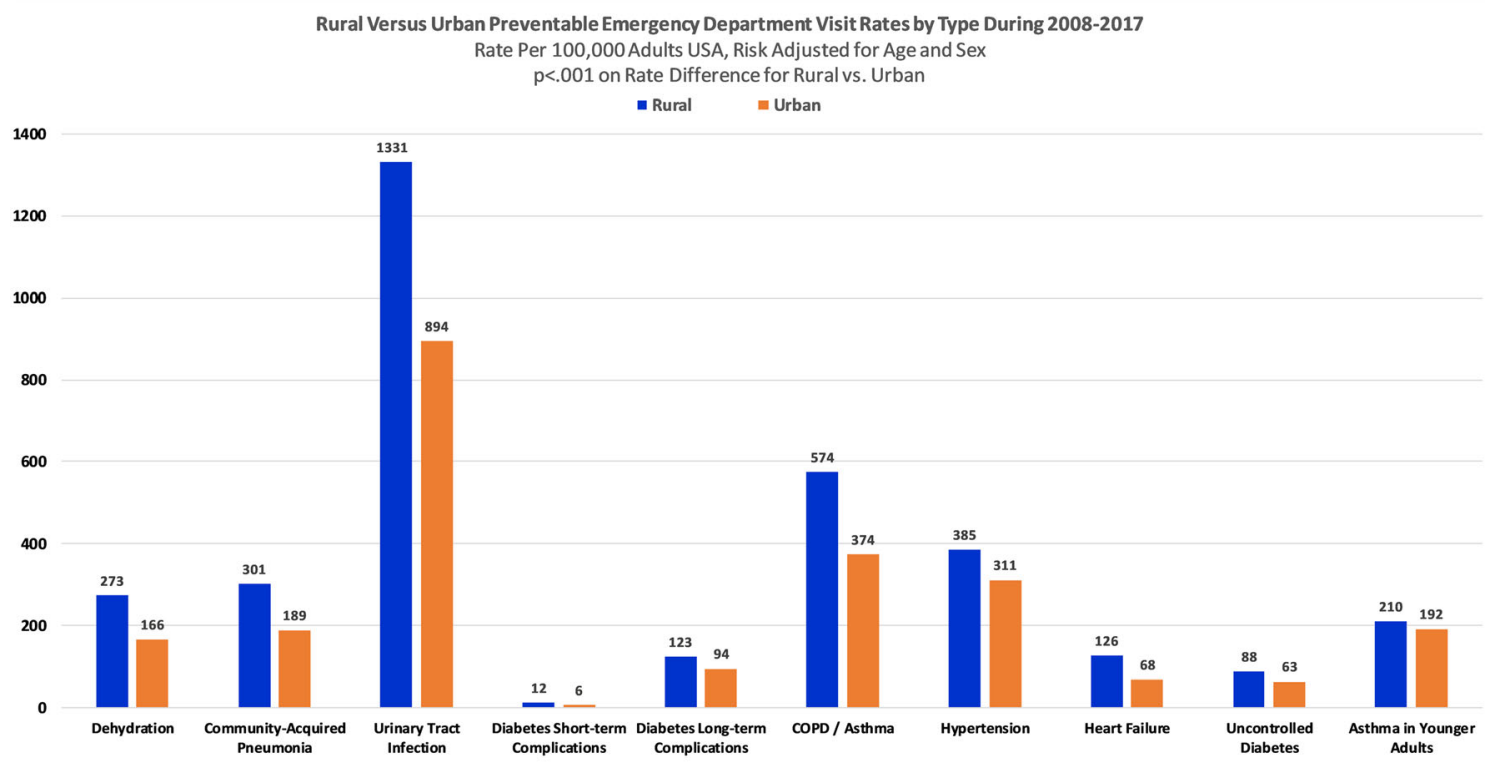

Figure 2 Rural versus urban preventable emergency department visit rates by condition during 2008-2017.

rule was for hospitalizations due to long-term complications of diabetes, hypertension, and asthma in younger adults, where urban rates moderately exceeded rural rates over the period. Preventable hospitalization rates increased the most for uncontrolled diabetes across all levels of rurality over the 10-year period; this was primarily due to the switch from the ICD-9CM to ICD-10-DM diagnosis coding system ( $p<.001$, Appendix Table 3).

In sensitivity analyses, results were similar when comparing unadjusted preventable hospitalization rates across all areas (Appendix Table 4).

\section{DISCUSSION}

Between 2008 and 2017, preventable ED visit rates and hospitalizations were substantially higher in rural than urban areas, driven by common conditions like pneumonia, COPD/asthma, dehydration, and urinary tract infections. Preventable $\mathrm{ED}$ visits increased more in rural versus urban residents, but the percentage increase was similar (30\% vs. $31 \%$ ) because rural residents started at a higher baseline. However, preventable hospitalizations decreased at a faster rate (22\% vs. $14 \%$ ) among rural versus urban residents over the decade. Thus, rural disparities in preventable acute care use are narrowing for preventable hospitalizations, but are substantial and have persisted over time for all acute care use.

Our results parallel prior research documenting falling hospitalization rates and rising ED visit rates nationally, as well as the higher rates of rural acute care use. ${ }^{13,21,31,32}$ However, we extend these prior findings to highlight differences between rural versus urban American adults in preventable hospitalization and $\mathrm{ED}$ visit rates.
There are at least three plausible reasons for the national trend we observed in all areas of declining preventable hospitalization rates and rising ED visit rates for visits that did not culminate in admission. First, this scenario may reflect a desirable tradeoff; with advances in lab diagnostics, for example, high-sensitivity troponin testing to rule out acute myocardial infarction, it is possible that ED visits that would previously have led to an admission can now be managed in the outpatient setting. ${ }^{33,34}$

Second, prior studies have observed a decline in all-cause and preventable hospitalizations coupled with a concomitant increase in observation stay ED visits for the same conditions, especially among the Medicare population. ${ }^{13,31,35,36}$ Observation stays are a mechanism by which patients remain on "outpatient status" despite being hospitalized and could somewhat artifactually create the appearance of a drop in admission rates when in fact it is simply a re-labeling of the same events. ${ }^{35}$

Third, efforts at delivery system and payment reform enacted by the Patient Protection and Affordable Care Act in 2010, particularly in Medicare, could be contributing to lower hospitalization rates. Medicare's Accountable Care Organization (ACO) programs during this time period, the Pioneer ACO Model and Medicare Shared Savings Program, and its outpatient value-based payment program, the Physician Value Modifier, all measured and rewarded providers' performance on preventable hospitalizations. ${ }^{37-39}$ Prior research indicates that these programs were associated with more timely outpatient care, better quality, and reduced inpatient utilization. ${ }^{38,40}$

Although the above reasons may explain the overall national trends we observed in this study, they still do not explain the large gap we find in preventable acute care use between rural versus urban Americans. There are at least three plausible reasons for the substantially higher rates of acute care use we find in rural areas. 
Table 2 A Comparison of Rural Versus Urban Preventable Hospitalization Rates at the Beginning and End of the Decade

\begin{tabular}{|c|c|c|c|c|c|c|c|c|}
\hline & \multicolumn{4}{|c|}{2008 rates per 100,000 adults $^{b}$} & \multicolumn{4}{|c|}{2017 rates per 100,000 adults $^{b}$} \\
\hline & Rural & Urban & Difference & $(95 \% \mathrm{CI})^{\mathrm{c}}$ & Rural & Urban & Difference & $(95 \% \mathrm{CI})^{\mathrm{c}}$ \\
\hline Adult population in millions, $N^{\mathrm{a}}$ & 38.9 & 191.1 & - & & 35.9 & 216.1 & - & \\
\hline All preventable hospitalizations & 2104 & 1666 & $439(434,443)$ & & 1634 & 1440 & $194(190,199)$ & \\
\hline Acute preventable hospitalizations & 930 & 659 & $270(267,273)$ & & 563 & 436 & $128(125,130)$ & \\
\hline Dehydration & 227 & 184 & $43(41,44)$ & & 157 & 153 & $4(3,5)$ & \\
\hline Community-acquired pneumonia & 484 & 294 & $190(188,192)$ & & 242 & 144 & $98(97,99)$ & \\
\hline Urinary tract infection & 218 & 181 & $37(36,39)$ & & 164 & 138 & $26(24,27)$ & \\
\hline Chronic preventable hospitalizations & 1175 & 1007 & $168(165,172)$ & & 1071 & 1004 & $67(63,70)$ & \\
\hline Diabetes short-term complications & 66 & 55 & $11(10,12)$ & & 68 & 58 & $11(10,11)$ & \\
\hline Diabetes long-term complications & 113 & 119 & $-6(-7,-5)$ & & 101 & 105 & $-3(-4,-2)$ & \\
\hline COPD/asthma & 471 & 337 & $134(132,136)$ & & 384 & 292 & $92(90,94)$ & \\
\hline Hypertension & 53 & 57 & $-4(-5,-3)$ & & 49 & 63 & $-14(-15,-13)$ & \\
\hline Heart failure & 417 & 388 & $29(27,31)$ & & 405 & 417 & $-12(-14-9)$ & \\
\hline Uncontrolled diabetes & 23 & 21 & $2(1,2)$ & & 48 & 52 & $-4(-5,-3)$ & \\
\hline Asthma in younger adults & 23 & 21 & $2(1,2)$ & & 9 & 12 & $-3(-3,-3)$ & \\
\hline Lower extremity amputation diabetes & 17 & 17 & $1(0,1)$ & & 31 & 29 & $1(1,2)$ & \\
\hline
\end{tabular}

${ }^{a}$ Denominator population supplied by AHRQ and derived from the US Census Bureau postcensal estimates summed over county populations for rural (not metropolitan area) and urban (city or metropolitan area $\geq 50,000$ population) counties

${ }^{b}$ Weighted estimates from the National Inpatient Sample (NIS) for all patients with at least 1 of the AHRQ Prevention Quality Indicator hospitalization. Using the NIS discharge weights to generate a national estimate by the rural and urban county categories above as noted for patient location on the discharge records. Rates were age-sex-adjusted using annual US Census data on the national US adult population with the direct method of standardization

${ }^{c} 95 \%$ confidence intervals (CIS) calculated using the normal approximation to the standard normal distribution

First, rural residents are sicker than urban residents. They are older, more likely to experience chronic conditions, disability, and complications of disease. ${ }^{3-5,8}$ In addition, rural Americans are more likely to be obese and use tobacco and are less likely to seek medical care when needed. ${ }^{5,41,42}$

Second, rural residents are much more likely to have multiple social risk factors, such as low income, lack of education, lack of employment, and poverty, than urban residents. ${ }^{2,12}$ These social risk factors are associated with higher preventable hospitalization and mortality rates. ${ }^{2}$

Third, and most importantly, rural residents have less access to primary care and specialist physicians in the ambulatory care setting than their urban counterparts. 1, 2, 6, 7 There are shortages of these physicians in rural areas during the time period we observed in this study. Furthermore, not only do rural residents have less access to ambulatory care, but the quality of care they receive is lower, especially for chronic conditions and preventive care. ${ }^{3,8}$ As a result, the higher rates of preventable acute care use we observed in rural residents most likely signal unmet demand for accessible, affordable, high-quality care in the ambulatory setting in rural areas.

Finally, it is important to address the differential changes in the rural versus urban trends we observed over the decade. In particular, why did preventable hospitalizations decrease more in rural than urban areas while preventable $E D$ visits grew at a higher absolute, and, similar percentage, rate? While our data do not allow us to test this directly, one plausible explanation is related to access to inpatient care. The past decade has seen a

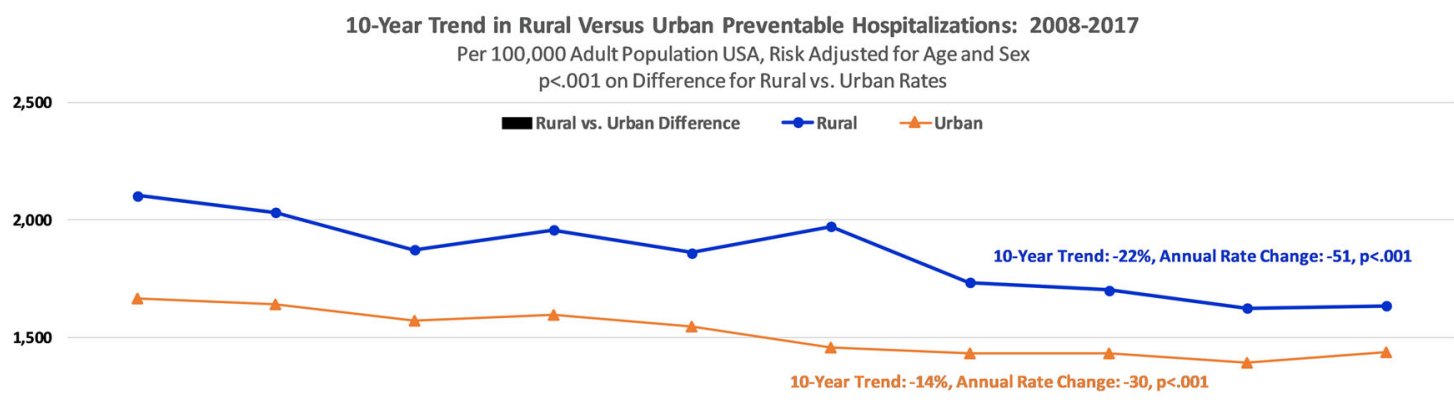

1,000

500

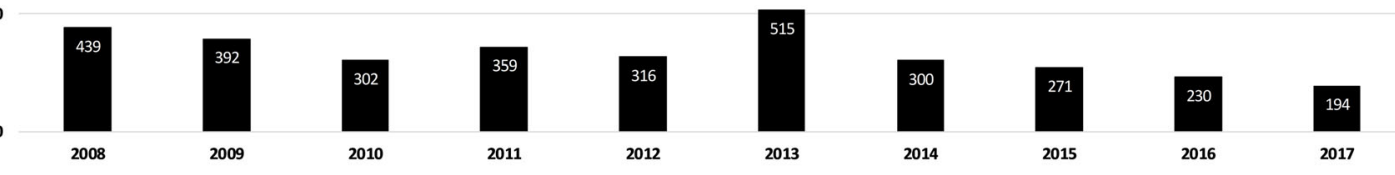

Figure 3 Ten-year trend in rural versus urban preventable hospitalizations: 2008-2017. 


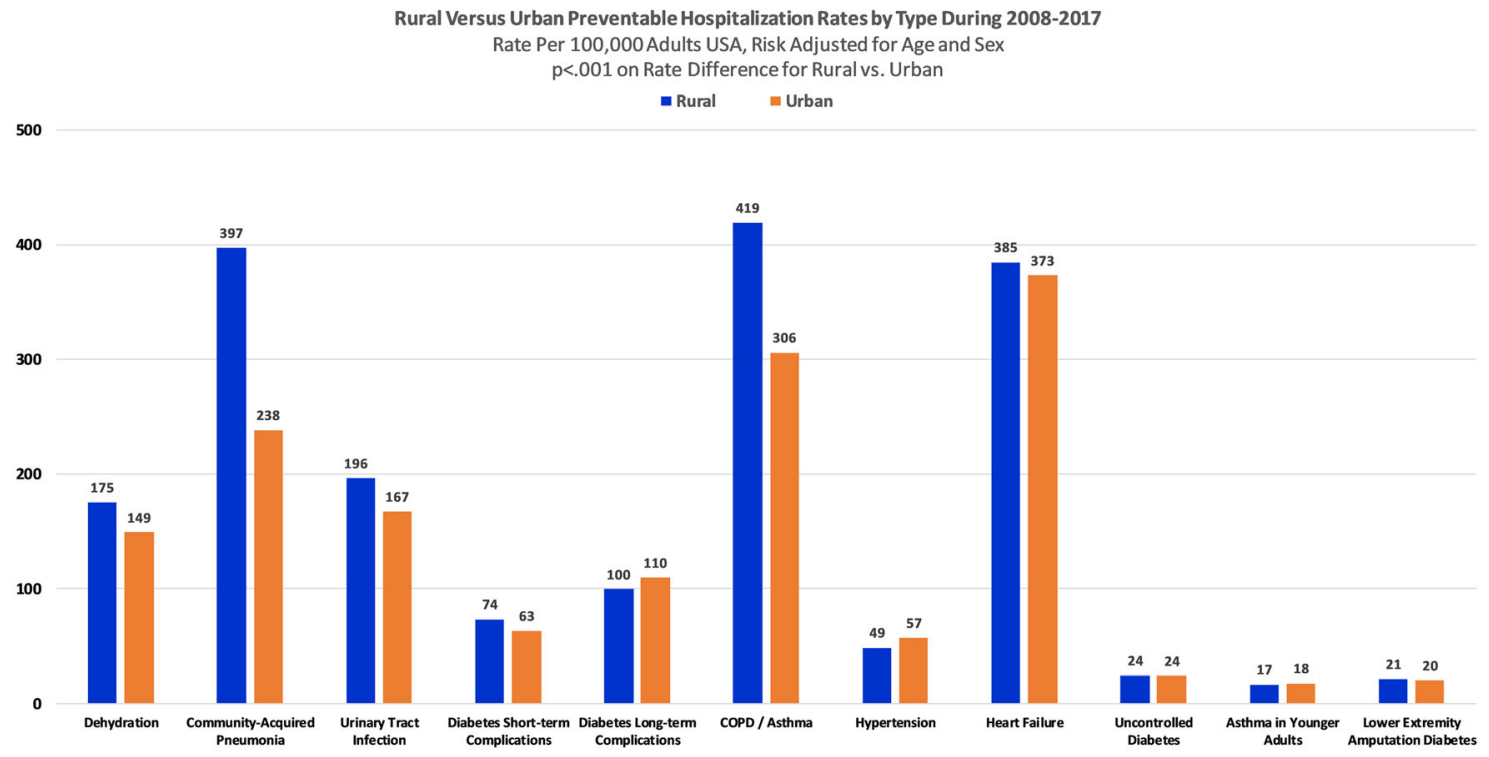

Figure 4 Rural versus urban preventable hospitalization rates by condition during 2008-2017.

much higher rate of hospital closures in rural versus urban areas, shrinking the pool of rural hospitals with the technological capacity to treat admitted patients with chronic and acute conditions. ${ }^{43}$ Some of the rural hospitals that closed inpatient services still provide urgent care and free-standing ED services. Although we did not study transfer rates, previous studies have shown much higher rates of transfer from the ED in rural versus urban hospitals, supporting the idea that rural residents presenting to EDs with concerning symptoms may be transferred to urban facilities if they warrant admission. ${ }^{44,45}$ Prior research also shows that rural hospital closures are associated with higher mortality rates among rural patients with chronic conditions. ${ }^{46}$ Lack of high-quality and timely inpatient care may help explain why the rural-urban mortality gap has been substantially widening over the past decade, with much higher rates of age-sex-adjusted rural versus urban mortality. ${ }^{47}$

Our study has limitations. This is an observational study, based on which we cannot make definitive causal inferences. Multiple changes in the insurance, delivery, and payment systems were implemented over the past decade, often in the same calendar years, making it difficult to pinpoint the effect of any single policy intervention. Additionally, even though the conditions we studied are purported to be potentially preventable through access to high-quality outpatient care, not all admissions for these conditions can actually be avoided. Particularly in older people, pneumonia or urinary tract infections can come on quickly, and may require hospitalization even if care is provided appropriately. Therefore, the feasible rate of these ED visits and admissions is not zero. However, the wide disparity between urban and rural individuals suggests ample room for improvement. In addition, we observed a very large uptick in preventable ED visits and hospitalizations for uncontrolled diabetes from 2014 to 2016 that was due to the switch from ICD-9-CM to ICD-10-CM codes. The ICD-10-CM codes for uncontrolled diabetes were used with much greater frequency than the previous ICD-9$\mathrm{CM}$ codes for the same condition. Thus, the uptick does not likely represent an actual increase in uncontrolled diabetes acute events in the underlying US adult population. However, the increase we observed exists in both the rural and urban categories; thus, it does not differentially impact our results with respect to comparison of trends. In addition, uncontrolled diabetes acute events only represent $3.5 \%$ of total preventable hospitalizations and $8.1 \%$ of preventable ED visits in 2017, so the effect on the aggregate trends is minimal.

In conclusion, we find that rural disparities in preventable hospitalizations are narrowing but are substantial and have persisted over time for all preventable acute care use, suggesting unmet demand for accessible, affordable, high-quality care in the ambulatory setting in rural areas.

Supplementary Information The online version contains supplementary material available at https://doi.org/10.1007/s11606-02006532-4.

Role of the Sponsor: Saint Louis University had no role in the design and conduct of the study; analysis, or interpretation of the data; preparation, review, or approval of the manuscript; and decision to submit the manuscript for publication.

Corresponding Author: Kenton J. Johnston, $\mathrm{PhD}$; Department of Health Management and Policy and Center for Outcomes Research, College for Public Health and Social Justice, Saint Louis University 3545 Lafayette Ave., Room 362, St. Louis, MO 63104, USA (e-mail: johnstonkj@slu.edu).

Author Contributions Dr. Johnston had full access to all the data in the study and takes responsibility for the integrity of the data and the accuracy of the data analysis.

Study concept and design: Johnston, Wen, Joynt Maddox Acquisition, analysis, or interpretation of data: all authors Drafting of the manuscript: Johnston, Joynt Maddox Critical revision of the manuscript for important intellectual content: all authors 
Statistical analysis: Johnston, Kotwal Obtained funding: Johnston

Administrative, technical, or material support: Johnston, Kotwal Study supervision: Johnston

Funding Saint Louis University purchased and provided access to the data used in this study.

Data Availability Available for purchase from the Agency for Healthcare Research and Quality (AHRQ) after execution of an approved data use agreement (see https://www.hcup-us.ahrq.gov/ databases.jsp for more details).

\section{Compliance with Ethical Standards:}

Conflict of Interest: Dr. Johnston receives grant funding from the National Institute on Aging (Grant No. R21AG065526). Dr. Joynt Maddox previously did work under contract with the US Department of Health and Human Services, Office of the Assistant Secretary for Planning and Evaluation, and receives grant funding from the National Heart, Lung, and Blood Institute (Grant No. RO1HL143421) and National Institute on Aging (Grant No. R01AG060935).

Statistical Code: Available to interested readers by contacting Dr. Kenton Johnston at kenton.johnston@slu.edu.

\section{REFERENCES}

1. Gong G, Phillips SG, Hudson C, Curti D, Philips BU. Higher US Rural Mortality Rates Linked To Socioeconomic Status, Physician Shortages, And Lack Of Health Insurance. Health Aff (Millwood). 2019;38(12):200310.

2. Johnston KJ, Wen H, Joynt Maddox KE. Lack Of Access To Specialists Associated With Mortality And Preventable Hospitalizations Of Rural Medicare Beneficiaries. Health Aff (Millwood). 2019;38(12):1993-2002.

3. Hale NL, Bennett KJ, Probst JC. Diabetes care and outcomes: Disparities across rural America. J Community Health. 2010;35(4):365-74.

4. Weeks WB, Kazis LE, Shen Y, Cong Z, Ren XS, Miller D, et al. Differences in health-related quality of life in rural and urban veterans. Am J Public Health. 2004;94(10):1762-7.

5. Jackson BE, Coultas DB, Suzuki S, Singh KP, Bae S. Rural-urban disparities in quality of life among patients with COPD. J Rural Health. 2013;29(SUPPL.1):1-14.

6. Goode AP, Freburger JK, Carey TS. The Influence of Rural Versus Urban Residence on Utilization and Receipt of Care for Chronic Low Back Pain. J Rural Health. 2013;29(2):205-14.

7. Jia H, Cowper DC, Tang Y, Litt E, Wilson L. Postacute Stroke Rehabilitation Utilization: Are There Differences Between Rural-Urban Patients and Taxonomies? J Rural Health. 2012;28(3):242-7.

8. Henning-Smith C, Kozhimannil K, Casey M, Prasad S, Moscovice I. Rural-Urban Differences in Medicare Quality Outcomes and the Impact of Risk Adjustment. Med Care. 2017 Sep;55(9):823-9.

9. Anderson AE, Henry KA, Samadder NJ, Merrill RM, Kinney AY. Rural vs urban residence affects risk-appropriate colorectal cancer screening. Clin Gastroenterol Hepatol Off Clin Pract J Am Gastroenterol Assoc. 2013 May;11(5):526-33.

10. Moy E, Garcia MC, Bastian B, Rossen LM, Ingram DD, Faul M, et al. Leading Causes of Death in Nonmetropolitan and Metropolitan AreasUnited States, 1999-2014. MMWR Surveill Summ. 2017;66(1):1-8.

11. Singh GK, Siahpush M. Widening rural-urban disparities in all-cause mortality and mortality from major causes of death in the USA, 19692009. J Urban Health. 2014;91(2):272-92.

12. Spencer JC, Wheeler SB, Rotter JS, Holmes GM. Decomposing Mortality Disparities in Urban and Rural U.S. Counties. Health Serv Res. 2018 Dec;53(6):4310-31.

13. Fingar KR, Barrett ML, Elixhauser A, Stocks C, Steiner C. Trends in Potentially Preventable Inpatient Hospital Admissions and Emergency Department Visits, Statistical Brief \# 195. Healthcare Cost and Utilization Project (HCUP) Statistical Briefs. 2015. p. 1-19.

14. Agency for Healthcare Research and Quality. Prevention Quality Indicators Overview [Internet]. 2020. Available from: https://www. qualityindicators.ahrq.gov/Modules/pqi_resources.aspx
15. Figueroa JF, Joynt Maddox KE, Beaulieu N, Wild RC, Jha AK. Concentration of Potentially Preventable Spending Among High-Cost Medicare Subpopulations. Ann Intern Med. 2017;167(10):706.

16. Muenchberger $\mathbf{H}$, Kendall E. Predictors of preventable hospitalization in chronic disease: priorities for change. $J$ Public Health Policy. 2010;31(2): 150-63.

17. Jiang H, Russo C, Barrett M. Nationwide Frequency and Costs of Potentially Preventable Hospitalizations, 2006: Statistical Brief \#72. [Internet]. Healthcare Cost and Utilization Project (HCUP) Statistical Briefs. 2009 [cited $2019 \mathrm{Feb}$ 8]. Available from: https://www.ncbi.nlm. nih.gov/books/NBK53971/

18. Galarraga JE, Mutter R, Pines JM. Costs associated with ambulatory care sensitive conditions across hospital-based settings. Acad Emerg Med. 2015;22(2):172-81.

19. Moore B, Stocks C, Owens P. Trends in Emergency Department Visits, 2006-2014. Healthcare Cost and Utilization Project (HCUP) Statistical Briefs. 2017. p. 1-19.

20. Freeman W, Weiss A, Heslin K. Overview of U.S. Hospital Stays in 2016: Variation by Geographic Region. Healthcare Cost and Utilization Project (HCUP) Statistical Briefs. 2018. p. 1-19.

21. Greenwood-Ericksen MB, Kocher K. Trends in Emergency Department Use by Rural and Urban Populations in the United States. JAMA Netw Open. 2019;2(4):e191919.

22. Owens PL, Barrett ML, Gibson TB, Andrews RM, Weinick RM, Mutter RL. Emergency department care in the United States: a profile of national data sources. Ann Emerg Med. 2010;56(2):150-65.

23. Healthcare Cost and Utilization Project. HCUP Nationwide Emergency Department Sample (NEDS) [Internet]. Rockville, MD: Agency for Healthcare Research and Quality; 2016 [cited 2016 Feb 26]. Available from: www.hcup-us.ahrq.gov/nedsoverview.jsp

24. Healthcare Cost and Utilization Project (HCUP). HCUP National Inpatient Sample (NIS) [Internet]. Rockville, MD: Agency for Healthcare Research and Quality; 2012 [cited 2017 Jan 26]. Available from: https://www. hcup-us.ahrq.gov/nisoverview.jsp

25. Agency for Healthcare Research and Quality. Population Denominator Data for Use with the HCUP Databases [Internet]. Healthcare Cost and Utilization Project. 2017 [cited 2020 Jun 11]. Available from: https:// www.hcup-us.ahrq.gov/reports/methods/2017-04.pdf

26. Agency for Healthcare Research and Quality. Guide to prevention quality indicators: hospital admission for ambulatory care sensitive conditions. In: AHRQ Quality Indicators. Version 3. Department of Health and Human Services; 2007. p. 1-59.

27. Davies S. Development of the Emergency Department Prevention Quality Indicators (ED PQI). In: AHRQ Annual Meeting. Stanford University Center for Primary Care and Outcomes Research; 2010. p. $1-28$.

28. National Center for Health Statistics. 2013 Urban-Rural Classification Scheme for Counties [Internet]. [cited 2020 Sep 25]. Available from: https://www.cdc.gov/nchs/data_access/urban_rural.htm\#2013_Urban-Rural_Classification_Scheme_for_Counties

29. National Center for Health Statistics. 2013 NCHS Urban-Rural Classification Scheme for Counties [Internet]. 2014 Apr [cited 2020 Sep 25]. (Series 2). Available from: https://www.cdc.gov/nchs/data/series/sr 02/sr02_166.pdf

30. Yaemsiri S, Alfier JM, Moy E, Rossen LM, Bastian B, Bolin J, et al. Healthy People 2020: Rural Areas Lag In Achieving Targets For Major Causes Of Death. Health Aff (Millwood). 2019;38(12):2027-31.

31. Figueroa JF, Burke LG, Zheng J, Orav EJ, Jha AK. Trends in Hospitalization vs Observation Stay for Ambulatory Care-Sensitive Conditions. JAMA Intern Med. 2019;179(12):1714.

32. Krumholz HM, Nuti SV, Downing NS, Normand S-LT, Wang Y. Mortality, Hospitalizations, and Expenditures for the Medicare Population Aged 65 Years or Older, 1999-2013. Jama. 2015;314(4):355.

33. Chew DP, Lambrakis K, Blyth A, Seshadri A, Edmonds MJR, Briffa T, et al. A Randomized Trial of a 1-Hour Troponin T Protocol in Suspected Acute Coronary Syndromes: The Rapid Assessment of Possible Acute Coronary Syndrome in the Emergency Department With High-Sensitivity Troponin T Study (RAPID-TnT). Circulation. 2019;140(19):1543-56.

34. Neumann JT, Twerenbold R, Ojeda F, Sörensen NA, Chapman AR, Shah ASV, et al. Application of High-Sensitivity Troponin in Suspected Myocardial Infarction. N Engl J Med. 2019;380(26):252940.

35. Hockenberry JM, Mutter R, Barrett M, Parlato J, Ross M A. Factors associated with prolonged observation services stays and the 
impact of long stays on patient cost. Health Serv Res. 2014;49:893909.

36. Ross MA, Hockenberry JM, Mutter R, Barrett M, Wheatley M, Pitts SR. Protocol-driven emergency department observation units offer savings, shorter stays, and reduced admissions. Health Aff Proj Hope. 2013;32(12):2149-56.

37. Pham HH, Cohen M, Conway PH. The Pioneer Accountable Care Organization Model. JAMA. 2014;312(16):1635.

38. Joynt Maddox KE, Epstein AM, Samson LW, Chen LM. Performance And Participation Of Physicians In Year One Of Medicare's Value-Based Payment Modifier Program. Health Aff Proj Hope. 2017;36(12):2175-84.

39. Centers for Medicare and Medicaid Services. Quality and Cost Measures Used in the Value Modifier. Value-Based Payment Modifier. 2018.

40. Nyweide DJ, Lee W, Cuerdon TT, Pham HH, Cox M, Rajkumar R, et al. Association of Pioneer Accountable Care Organizations vs Traditional Medicare Fee for Service With Spending, Utilization, and Patient Experience. JAMA. 2015;313(21):2152-61.

41. Atkins GT, Kim T, Munson J. Residence in rural areas of the United States and lung cancer mortality: Disease incidence, treatment disparities, and stage-specific survival. Ann Am Thorac Soc. 2017; 14(3):403-11
42. Hales CM, Fryar CD, Carroll MD, Freedman DS, Aoki Y, Ogden CL. Differences in obesity prevalence by demographic characteristics and urbanization level among adults in the United States, 2013-2016. JAMA J Am Med Assoc. 2018;319(23):2419-29.

43. Frakt AB. The Rural Hospital Problem. JAMA. 2019 Jun 18;321(23):2271.

44. Bennett KJ, Probst JC, Bullard JC, Crouch E. The Importance of Rural Hospitals: Transfers and 30-day Readmissions Among Rural Residents and Patients Presenting at Rural Hospitals. Popul Health Manag. 2019 Apr;22(2):120-6.

45. Lyon M, Sturgis L, Lendermon D, Kuchinski AM, Mueller T, Loeffler P, et al. Rural ED transfers due to lack of radiology services. Am J Emerg Med. 2015;33(11):1630-4.

46. Gujral K, Basu A. Impact of Rural and Urban Hospital Closures on Inpatient Mortality [Internet]. Cambridge, MA: National Bureau of Economic Research; 2019 Aug [cited 2020 Oct 21] p. w26182. Report No.: w26182. Available from: http://www.nber.org/papers/w26182.pdf

47. Cosby AG, McDoom-Echebiri MM, James W, Khandekar H, Brown W, Hanna HL. Growth and Persistence of Place-Based Mortality in the United States: The Rural Mortality Penalty. Am J Public Health. 2019 Jan;109(1):155-62.

Publisher's Note: Springer Nature remains neutral with regard to jurisdictional claims in published maps and institutional affiliations. 